\title{
Low-dose midazolam infusion for oculoplastic surgery under local anaesthesia
}

\begin{abstract}
Purpose Oculoplastic surgery with infiltration of local anaesthesia at the operative site performed as a day-case procedure is both efficient and cost-effective. Patients considered unsuitable for this because of fear or apprehension may, however, benefit from per-operative conscious sedation. We sought to study the efficacy and safety of this using midazolam, a water-soluble benzodiazepine. Method We have performed a controlled clinical trial comparing the effect of a lowdose intravenous infusion of midazolam $(0.2 \mathrm{mg} / \mathrm{ml}$ of normal saline at a rate of $1 \mathrm{mg} / \mathrm{h}$ ) with saline placebo on 48 subjects undergoing oculoplastic surgery with local anaesthesia. Patients were given pre- and post-operative questionnaires assessing, amongst other factors, anxiety levels, pain, degree of reported amnesia and psychomotor recovery. Results Using the low-dose midazolam infusion no adverse cardiorespiratory reactions occurred. Patients receiving midazolam reported remembering significantly less about their operation than controls $(p=0.04)$ and showed significantly lower state-anxiety after their operation than before $(p<0.02)$. This change was not noted in the placebo group. There was no significant difference in the psychomotor performance of patients given midazolam compared with controls $2 \mathrm{~h}$ after surgery.

Conclusions A low-dose continuous infusion of midazolam can be used to safely provide effective anxiolysis and conscious sedation with good psychomotor recovery during oculoplastic procedures in a day-case setting.
\end{abstract}

Key words Amnesia, Anaesthesia, Midazolam, Oculoplastic surgery, Psychomotor performance, State-anxiety

Most minor eyelid surgery is performed under infiltration of local anaesthetic at the operative site. ${ }^{1}$ Local anaesthesia should last long enough for the procedure to be performed with minimal discomfort to the patient. More major eyelid and reconstructive surgery, however, requires a
S. BISWAS, M. BHATNAGAR,

M. RHATIGAN, J. KINCEY, R. SLATER,

B. LEATHERBARROW

longer operative time and may therefore be unsuited to local anaesthesia. In addition, many patients are not considered suitable for local anaesthesia because of their apprehension or anxiety. General anaesthesia may be acceptable in a large proportion of cases but requires more time and may increase the morbidity associated with the procedure. ${ }^{2,3}$ The option of local anaesthesia with per-operative sedation to overcome some of these problems is an alternative. Such sedation should ideally provide relief of anxiety and amnesia while maintaining consciousness with full patient cooperation. $^{4}$

Midazolam is a water-soluble benzodiazepine of the imidazobenzodiazepine class. It has powerful hypnotic, anxiolytic and anterograde amnestic properties and, when given as a premedicant, provides good patient co-operation for many procedures. It is painless on intravenous injection and readily crosses the blood-brain barrier producing a quick onset of action. Its additional property of having a large volume of distribution and rapid elimination half-life $\left(t_{1 / 2}=1.5-3.5 \mathrm{~h}\right)$ results in a short recovery period. ${ }^{5}$

We report a controlled clinical trial investigating the safety and efficacy of a peroperative, continuous intravenous low-dose infusion of midazolam to provide conscious sedation during oculoplastic surgery. We measured the patients' levels of anxiety and pain, their subjective amnesia and their general satisfaction with the procedure. The psychomotor recovery after the procedure was assessed using a Digital Symbol Substitution Test as a test of visuo-motor function.

\section{Materials and methods}

After obtaining ethics committee approval, 55 patients of either sex who were considered suitable for day-case surgery under local anaesthesia were selected at random. The age range was $16-85$ years. Patients with previous cardiorespiratory disease, hepatic or renal disease, a history of drug abuse, pregnancy or existing use of sedative or psychotropic drugs were excluded. Informed consent was required
S. Biswas

M. Bhatnagar

M. Rhatigan

B. Leatherbarrow

Manchester Royal Eye

Hospital

Manchester, UK

J. Kincey

Department of Health

Psychology

Central Manchester

Healthcare Trust

Manchester, UK

R. Slater

Department of Anaesthesia Manchester Royal Infirmary Manchester, UK

Mr S. Biswas, FRCOphth Manchester Royal Eye

Hospital

Oxford Road

Manchester M13 9WH,UK

Tel: +44(0)1612761234

Fax: +44 (0)1612726618

Received:

22 September 1998

Accepted in revised form:

11 December 1998 
Table 1. Surgical procedure by groups

\begin{tabular}{|c|c|c|}
\hline Procedure & $\begin{array}{l}\text { Midazolam } \\
\text { group }(n=24)\end{array}$ & $\begin{array}{l}\text { Control group } \\
\quad(n=24)\end{array}$ \\
\hline $\begin{array}{l}\text { Lower eyelid entropion/ectropion } \\
\text { repair }\end{array}$ & 7 & 5 \\
\hline Full-thickness skin graft & 4 & 2 \\
\hline $\begin{array}{l}\text { Upper and lower eyelid } \\
\text { reconstruction after Mohs } \\
\text { surgery }\end{array}$ & 3 & 5 \\
\hline \multicolumn{3}{|l|}{ Micrographic surgery } \\
\hline Blepharoplasty & 1 & 1 \\
\hline Lateral tarsorrhaphy & 1 & 3 \\
\hline Ptosis repair & 4 & 4 \\
\hline $\begin{array}{l}\text { Anterior lamellar repositioning for } \\
\text { upper eyelid ectropion }\end{array}$ & 2 & 1 \\
\hline Wedge excision biopsy & 1 & 2 \\
\hline $\begin{array}{l}\text { Drilling of hydroxyapatite orbital } \\
\text { implant }\end{array}$ & 1 & 1 \\
\hline
\end{tabular}

for inclusion in the study, after which 48 patients agreed to take part. The patients were randomly allocated to receive either a placebo $(0.9 \%$ normal saline $)$ or midazolam. The patients were masked as to which protocol they received. Midazolam, at a strength of $0.2 \mathrm{mg} / \mathrm{ml}$ of normal saline, was delivered by means of an intravenous infusion via a syringe driver (Graseby 3100, Graseby Medical, Watford, Herts, UK). A bolus dose of $0.5 \mathrm{mg}$ of midazolam was given $1 \mathrm{~min}$ prior to the infiltration of the operative site with local anaesthesia.

Thereafter a continuous infusion of midazolam at a rate of $1 \mathrm{mg} / \mathrm{h}$ was maintained until $5 \mathrm{~min}$ before the end of the operation, whereupon the infusion was discontinued. The same delivery protocol was used for patients receiving placebo.

Local anaesthesia was administered by means of a subcutaneous infiltration of a 50:50 mixture of lignocaine $2 \%$ and bupivacaine $0.5 \%$ with adrenaline 1:200 000 . Oxygen saturation with a pulse oximeter (Ohmeda Biox 3700, Louisville, CO 80027), electrocardiograms and blood pressure were monitored continuously (Simonsen \& Weel ECG triscope, Albertslund, Denmark).

Monitoring was performed peri-operatively by an anaesthetic-trained nurse. Verbal contact was maintained with the patient at all times. Patients were prepared and draped for surgery to allow full exposure of the face, thus enabling the patient to breath room air throughout the procedure. Oxygen, delivered by a nasal speculum, was available if the patient's oxygen saturation fell below $90 \%$. The types of operation performed are listed in Table 1.

Table 2. Six-item short form of the state scale of the Spielberger StateTrait Anxiety Inventory (Marteau and Bekker ${ }^{6}$ )

$\begin{array}{ll}\text { 1. } & \text { I feel calm } \\ 2 . & \text { I feel tense } \\ \text { 3. } & \text { I feel upset } \\ \text { 4. } & \text { I feel relaxed } \\ 5 . & \text { I feel content } \\ 6 . & \text { I feel worried }\end{array}$

Scores: not at all $=1$, somewhat $=2$, moderately $=3$, very much $=4$ (with reversed scoring where appropriate).
Table 3. Self-evaluation questionnaire

1. How painful was the operation?

2. How much can you remember about your operation?

3. How much do you feel you understood about your operation?

4. How satisfied are you with what you were told about your operation?

5. Imagine you could decide again whether or not to have the operation. Think about what you know now. Would you have it again?

6. Do you feel you were kept in the overall picture? (Yes/No)

Answers were graded 0 to 100 except for the last question.

The following psychological parameters were tested:

1. Anxiety assessment: Six-item short form of the state scale of the Spielberger State-Trait Anxiety Inventory (STAI), ${ }^{6}$ pre-operatively and $1 \mathrm{~h}$ post-operatively (Table 2 ).

2. Pain, amnesia, understanding of the surgical procedure and willingness to undergo the procedure again: self-evaluation questionnaire $1 \mathrm{~h}$ post-operatively (Table 3 ).

3. Psychomotor recovery: Digital Symbol Substitution Test (DSST) from the Wechsler Adult Intelligence Scale (WAIS), $2 \mathrm{~h}$ post-operatively.

A number of statistical methods were used, including the Kolmogorov-Smirnov goodness of fit test to determine normality for all the collected variables. Normally distributed, continuous data (e.g. age, DSST and operation time) were subjected to $t$-tests for independent samples. Non-parametric tests were carried out for data not normally distributed (pre-operative, post-operative and self-evaluation questionnaires). Data were cross-tabulated and the following tests were conducted: Fisher's Exact test, the Mann-Whitney $U$-test, Wilcoxon matched-pairs signed ranks test. A $p$ value less than 0.05 was considered to be statistically significant.

\section{Results}

Of the 55 patients approached to participate in the study 5 patients declined and 2 observations were discarded due to incomplete data. This left 48 participants. The average total dose of midazolam given was $1.66 \mathrm{mg}$ ( \pm $0.54 \mathrm{mg}$ ). No episodes of hypoxia, as indicated by oxygen saturation falling below $90 \%$, or airway obstruction were observed in either group and no patient required supplementary oxygen. There were no cardiovascular complications and no episodes of acute hypotension or hypertension, defined as a fall or rise in mean arterial pressure greater than $30 \%$ from baseline respectively, in either group. Table 4 shows the mean age and operative times, as well as the DSST and pre- and post-operative state-anxiety mean scores. No significant difference existed between the mean age or operative times recorded in either group.

The DSST results revealed a high score for the midazolam group compared with the controls (40.2 \pm 13.0 vs $33.7 \pm 12.3$ respectively; $p=0.08$ ). Though this was not a significant difference, a trend existed. 
Table 4. Mean ( $\pm S D$ ) age and operation times, Digital Symbol Substitution Test (DSST), and pre-and post-operative state anxiety mean scores

\begin{tabular}{lccccc}
\hline Group & Age (years) & Operation time $(\mathrm{min})$ & DSST & $\begin{array}{c}\text { Pre-operative } \\
\text { state-anxiety }\end{array}$ & $\begin{array}{c}\text { Post-operative } \\
\text { state-anxiety }\end{array}$ \\
\hline Midazolam $(n=24)$ & $64.8(15.2)$ & $75.1(32.5)$ & $40.2(13.0)$ & 17.33 & $16.17^{*}$ \\
Control $(n=24)$ & $62.5(18.2)$ & $61.5(24.3)$ & $33.7(12.3)$ & 16.88 & 16.38 \\
\hline
\end{tabular}

*Wilcoxon matched pairs signed ranks test, $p=0.02$

The Spielberger State-Trait Anxiety Inventory revealed a significant reduction in the mean overall postoperative scores compared with the mean pre-operative scores in the midazolam group (mean pre-operative score $=17.33$ vs mean post-operative score $=16.17 ; p=$ $0.03)$. There was no significant reduction in state-anxiety for the control group of patients (mean pre-operative score $=16.88$ vs mean post-operative score $=16.38$ ).

The self-evaluation questionnaire revealed a significant difference in how much the patient reported remembering their operation between the two groups (Table 5). The group receiving midazolam reported remembering significantly less than the placebo group $(p=0.04)$. Although no significant differences were noted between the two groups as regards the other individual items, an overall differing trend was noted. The patients' perception of pain tended to be less in the midazolam group and they tended to have a greater understanding about their operation with greater satisfaction regarding what they were told about their operation as well as an increased willingness to undergo the procedure again.

\section{Discussion}

The anxiolytic properties of midazolam were confirmed in this study as borne out by the shift in the STAI scores seen in the midazolam group. Thus patients, feeling more comfortable during surgery, may be able to tolerate lengthier procedures. Reduced anxiety may also make patients less concerned about pain, reducing their requirement for supplementary per-operative local anaesthetic injections.

Patients in the midazolam group remembered significantly less about their operation than the control group, presumably as a consequence of midazolam's known amnestic properties. No patient reported total amnesia but one patient reported experiencing $98 \%$ anterograde amnesia. Studies have shown that when given as a premedicant for conscious sedation in patients undergoing various endoscopic procedures, cardiac catheterisation and spinal anaesthesia, midazolam induced a greater degree of amnesia than diazepam. ${ }^{5}$ The

Table 5. Self-evaluation questionnaire scores (out of 100)

\begin{tabular}{lcc}
\hline Question & $\begin{array}{c}\text { Midazolam } \\
(n=24)\end{array}$ & $\begin{array}{c}\text { Placebo } \\
(n=24)\end{array}$ \\
\hline 1. How painful? & 14.5 & 19.6 \\
2. How much remembered? & 67.5 & $79.2^{*}$ \\
3. How much understood? & 74.8 & 66.9 \\
4. How satisfied? & 78.8 & 73.2 \\
5. Would have operation again? & 76.6 & 68.7 \\
\hline
\end{tabular}

*Mann-Whitney $U$-test, $p<0.05$. importance of amnesia is well appreciated as it is known that decreased recall of events may improve the patient's tolerance to surgical procedures ${ }^{6,7}$ and may benefit postoperative recovery. ${ }^{8}$

Psychomotor recovery, as measured by the DSST, was greater in the midazolam group than the control group. Though this was not significant, a trend existed. The reason for this could be pre-existing difference between the two groups. A pre-operative DSST could have examined this possibility. The alternative explanation is that a larger sample size might have confirmed the significance of this trend. However, considering the low doses of midazolam used, it is likely that the sedative effect of midazolam wore off rapidly after its discontinuation. In the midazolam group there was no significant correlation of psychomotor recovery with age or duration of operation. In the control group, however, there was a significant correlation of both age and duration of procedure with psychomotor recovery ( $r=-0.69, p<0.001 ; r=0.61 ; p<0.001$, respectively). Whilst again this may be due to a pre-operative difference between the groups, midazolam may even out the visuomotor performance across the age groups and lessen the impact of surgical duration. At doses of $0.07-0.1 \mathrm{mg} / \mathrm{kg}$ psychomotor function is affected for 3-4 h after intravenous administration of midazolam. ${ }^{5}$ Our study has shown that, at the low dose used, patients' psychomotor recovery was as good as controls and these patients were fit and alert enough to be discharged from the day surgery unit in the presence of an accompanying adult. Some groups have recommended the use of flumazenil to reverse midazolam-induced sedation. ${ }^{7}$ We felt that this was unnecessary.

Sedation is widely used in many fields of surgery including oculoplastic surgery. ${ }^{9}$ Various agents such as propofol, barbiturates, benzodiazepines and opiates or a combination of these have been used. ${ }^{4,5,10}$ Desired clinical effects from these various agents include relaxation and relief of anxiety, co-operation and amnesia. These agents do not provide anaesthesia, but may through their anxiolytic activity increase the patient's comfort throughout an operative procedure as well as reducing post-operative anxiety. Per-operative sedation has certain advantages over premedication in terms of the degree of control one can achieve through giving it via an intravenous route. Onset of sedation is rapid when given intravenously compared with an oral or intramuscular route and maintenance of this sedation is easier. It is often difficult to administer oral sedation such that it would reach peak effect at the onset of surgery and maintenance of sufficient sedation is not guaranteed. ${ }^{11}$ 
The dose of midazolam in this study was not titrated according to the age or weight of the patients; instead a low dose of sedative was given via a slow infusion as a set protocol with constant monitoring throughout the procedure. Such safety features enabled the sedation to be given with the supervision of a trained anaesthetic nurse but without the requirement for an anaesthetist. Although the sedative doses were low they still provided effective anxiolysis and amnesia throughout the surgical procedure.

\section{Conclusions}

For many patients undergoing oculoplastic and reconstructive surgery the option for general anaesthesia may be less desirable than local anaesthesia because of increased risk of morbidity. In addition, in certain circumstances, local anaesthesia may assist patient cooperation per-operatively and thus facilitate the surgical outcome. Local anaesthesia with conscious sedation provides an alternative that more patients can tolerate. Conscious sedation should aim to provide anxiolysis, relaxation, amnesia and co-operation. Midazolam is well tolerated as an intravenous infusion and has a good safety record. This study has shown that its administration in a low-dose continuous infusion is safe, and provides effective amnesia, anxiolysis and patient satisfaction for oculoplastic procedures. It has also shown that by $2 \mathrm{~h}$ after the end of the procedure patients can be safely discharged home.
The authors would like to thank Dr Val Hillier, Department of Medical Computation, University of Manchester, for her assistance with the statistical analysis.

\section{References}

1. Wong D, Hunter JM, Mostafa SM. In: Mostafa SM, editor. Anaesthesia for ophthalmic surgery. Oxford: Oxford Medical Publications, 1991:250-75.

2. Smith BL, Young PN. Day stay anaesthesia: a follow-up of day patients undergoing dental operations under general anaesthesia with tracheal intubation. Anaesthesia 1976;31:181-9.

3. Routh GS. Day care surgery under general anaesthesia in a purpose built unit. Anaesthesia 1979;34:809-11.

4. Loeffler PM. Oral benzodiazepines and conscious sedation: a review. J Oral Maxillofac Surg 1992;50:989-97.

5. Dundee JW, Halliday NJ, Harper KW, Brogden RN. Midazolam: a review of its pharmacological properties and therapeutic use. Drugs 1984;28:519-43.

6. Marteau T, Bekker H. The development of a 6 item shortform of the state scale of the Spielberger State Trait Anxiety Inventory (STAI). Br J Clin Psychol 1992;31:301-6.

7. Birch BRP, Anson KM, Clifford E, Miller RA. Day-case surgery: enhanced recovery with flumazenil. J R Soc Med 1990;83:436-8.

8. O'Boyle CA. Benzodiazepine-induced amnesia and anaesthetic practice: a review. Psychopharmacol ser. 1988;6:146-65.

9. Bartamian M, Meyer DR. Site of service, anaesthesia and post-operative practice pattern for oculoplastic and orbital surgeries. Ophthalmology 1996;103:1628-33.

10. Fulton B, Sorkin EM. Propofol: an overview of its pharmacology and a review of its clinical efficacy in intensive care sedation. Drugs 1995;50:636-57.

11. Moore PA, Ramsay DS, Finder RL, et al. Pharmacological modalities in the management and treatment of dental anxiety. Dent Clin North Am 1988;32:803. 